\title{
S'établir en agriculture sans prendre la relève : un sentier parsemé d'embûches
}

\author{
Diane Parent \\ Université Laval
}

\section{Une population agricole en panne de renouvellement}

Jusqu'à maintenant au Québec, comme dans l'ensemble nord-américain, le renouvellement des agriculteurs s'est fait massivement de l'intérieur : il y avait une hérédité du métier due, entre autres, à la pression de la logique patrimoniale et à la difficulté d'accéder à la propriété pour ceux qui n'ont pas de famille derrière eux.

À première vue, la question de la relève agricole ne semble pas être un problème de nombre d'intéressés, mais plutôt d'accès à la propriété, surtout pour ceux qui s'établissent hors du cadre familial. Mais cette nouvelle relève, hier encore marginale, est devenue incontournable pour le devenir de l'agriculture. Elle est cependant mal connue et peu reconnue aux plans socioprofessionnel et politique.

Or, pour freiner le déclin du nombre de fermes, l'agriculture ne peut faire l'économie de cette population (tableau 1). En effet, selon le dernier recensement agricole, on constate qu'au Québec le nombre de fermes n'a cessé de décliner, subissant une baisse de $4 \%$ par rapport à 2001 et se situant à moins de 30700 . On note aussi que «la population agricole a diminué de $26 \%$ entre 1991 et 2001 , pour ne plus représenter que $6,4 \%$ de la population rurale et $1,3 \%$ de la population québécoise $»^{1}$.

Tableau 1 - Évolution de la population agricole (\%) par tranche d'âge (2001-2006)

\begin{tabular}{rcc} 
& Canada & Québec \\
\cline { 2 - 3 } 35 ans et moins & $-33,41$ & $-26,26$ \\
$35-55$ ans & $-13,05$ & $-11,62$ \\
55 ans et plus & 9,22 & 16,51 \\
\hline Nombre total d'agriculteurs (2006) & 327000 & 45470 \\
\hline Âge Moyen (2006) & $(-5,5 \%$ par rapport à 2001) & $(-4 \%$ par rapport à 2001) \\
\hline Source : Statistique Canada $(2006)^{2}$ & 52 & 49
\end{tabular}

Comme dans l'ensemble de la société québécoise, la population agricole est de plus en plus vieillissante, mais à un rythme plus rapide. Le taux de renouvellement des générations, c'est-à-dire le nombre d'agriculteurs de moins de 35 ans, par rapport à ceux de plus de 55 ans, est en chute libre. Si, en 1991, ce taux était de $112 \%$, c'est-à-dire 1,12 agriculteur de moins de 35 ans pour 1 agriculteur de plus de 55 ans, il est aujourd'hui de $35 \%$ (tableau 2), ce qui signifie que seulement 1 agriculteur sur 3 est remplacé. Rappelons par ailleurs que le ministère québécois de l'Agriculture a évalué à 1000 le nombre d'établissements nécessaires pour maintenir le nombre actuel de fermes, alors qu'effectivement il ne s'en réalise que de 600 à $700^{3}$.

Contrairement à l'établissement par voie de transfert familial, peu d'études ont documenté chez nous le phénomène d'installation hors de la sphère familiale et ce, contrairement à la France par exemple ${ }^{4}$. Dans le 
but d'identifier les caractéristiques de l'installation hors cadre familial et de relever les facteurs caractérisant les parcours de succès et d'insuccès, nous som- mes allés à la rencontre de 57 d'entre eux en 2003$2004^{5}$.

Tableau 2 - Taux de renouvellement des générations en agriculture (\%)

\begin{tabular}{lcc} 
Année & Canada & Québec \\
\hline 1991 & 62 & 112 \\
1996 & 49 & 89 \\
2001 & 30 & 53 \\
$\mathbf{2 0 0 6}$ & 23 & 35
\end{tabular}

Source : Traget Laval adapté du recensement agricole de Statistique Canada (2006)

\section{Qu'est-ce que la relève hors cadre familial ?}

Nous avons défini la relève hors cadre familial comme étant tout individu âgé entre 18 et 40 ans qui s'est établi sur une exploitation agricole acquise d'un vendeur avec lequel il n'avait aucun lien de parenté. Nos répondants pouvaient cependant être originaires du milieu agricole. Nous avons atteint la saturation des informations recherchées après avoir réalisé 57 entrevues en profondeur auprès d'exploitants établis entre 1997 et 2000 (31 hommes et 26 femmes) et regroupés au sein de 30 exploitations agricoles dont $80 \%$ ont le statut juridique de co-exploitation entre conjoints avec un partage des parts avoisinant ou égalant 50/50 entre les participants. Cet échantillon regroupait 20 entreprises encore actives, 7 qui avaient cessé leurs activités et 3 qui étaient en grande difficulté financière. Fait à noter à propos de la relève non familiale, ils sont plus âgés que la relève familiale (31 ans contre 26 ans) et la majorité exploite en couple en ayant démarré une production originale, dans des secteurs d'activité très variés mais reconnus tout de même comme étant en émergence ou non traditionnels, c'est-à-dire autres que le lait, le porc, le bovin de boucherie et la volaille.

La grille d'entretien avait été conçue de manière à faire ressortir les variables en lien avec le succès ou l'insuccès à l'établissement et que nous avions déjà identifiées dans nos recherches sur la relève familiale ${ }^{6}$ comme les facteurs déterminants, les éléments les plus facilitants, la nature des obstacles rencontrés et ce, afin de tracer les types de trajectoires suivies par les répondants. Comme le démontre le tableau 3, les unités d'analyse retenues sont les antécédents du répondant, le processus de l'établissement, le projet, l'établissement comme tel, le bilan de leur expérience de même que les perspectives d'avenir.

\section{Les deux étapes de l'établissement : pré-démarrage et démarrage}

L'analyse des facteurs réunis fait prendre conscience du cheminement d'une idée pour réaliser un vieux rêve qui mijote depuis l'adolescence, voire l'enfance. Le poids des antécédents sur l'établissement par rapport aux étapes préparatoires est important et permet d'avancer que nos répondants n'ont pas démarré leur projet sur un coup de tête. En effet, chez plus de trois entreprises sur quatre, il s'agit de la poursuite d'une idée ancrée dès l'adolescence et dont la réalisation a fait l'objet d'une préparation réfléchie marquée par la présence d'au moins deux étapes préparatoires sur trois (formation, expériences antérieures et autres préparations telles stages, parrainages ou socialisation dès l'enfance). Cependant, force est de constater que les entreprises en difficulté et les échecs présentent un score plus faible. Par exemple, trois entreprises se sont lancées sans formation initiale et en n'ayant réalisé qu'une seule étape : dans le premier cas, l'un des co-exploitants avait une expérience de travailleur agricole dans la production démarrée. Dans les deux autres cas, les co-exploitants avaient réalisé un stage ou fait des lectures et tenté quelques expérimentations.

Le processus d'établissement s'est déroulé en deux grands moments et correspond à celui vécu dans les établissements d'entreprises des autres secteurs de l'économie, soit une période de pré-démarrage et une 
période de démarrage proprement dite. Il ressort que le pré-démarrage, qui correspond à l'émergence de l'idée jusqu'à sa concrétisation, varie entre 1 mois et 3 ans, s'établissant à 13 mois en moyenne. Le mo- dèle de démarrage choisi par plus des trois quarts des entreprises est celui de l'acquisition de fermes dès le départ ou dans les premières années de l'entreprise, le tout complété par la location d'une partie du foncier.

\section{Tableau 3 - Unités d'analyses et indicateurs identifiés pour analyser le parcours d'établissement}

\begin{tabular}{|c|c|}
\hline Unités d'analyse & Indicateurs \\
\hline Les antécédents & $\begin{array}{l}\text { Caractéristiques personnelles (liées au profil entrepreneurial) } \\
\text { Bagage de connaissances, des compétences, des expériences } \\
\text { Valeurs personnelles } \\
\text { Réseaux familial, social et professionnel }\end{array}$ \\
\hline $\begin{array}{l}\text { Le processus } \\
\text { d'établissement }\end{array}$ & $\begin{array}{l}\text { Grands moments } \\
\text { Démarches } \\
\text { Rôles des coexploitants, s'il y a lieu } \\
\text { Éléments déclencheurs } \\
\text { Retards, blocages }\end{array}$ \\
\hline Projet d'établissement & $\begin{array}{l}\text { Projet initial et vision } \\
\text { Soutien réseau personnel } \\
\text { Soutien des intervenants agricoles ou autres } \\
\text { Soutien du vendeur ou locateur ou intégrateur } \\
\text { Éléments de contexte agricole } \\
\text { Dynamique des coexploitants, s'il y a lieu }\end{array}$ \\
\hline L'établissement & $\begin{array}{l}\text { Évolution du projet } \\
\text { Acquis de connaissances } \\
\text { Poids du réseau personnel } \\
\text { Apport des intervenants agricoles } \\
\text { Soutien du vendeur ou locateur ou intégrateur, s'il y a lieu } \\
\text { Éléments de contexte du milieu agricole } \\
\text { Poids de la dynamique entre coexploitants, s'il y a lieu }\end{array}$ \\
\hline $\begin{array}{l}\text { Bilan et perspectives } \\
\text { d'avenir }\end{array}$ & $\begin{array}{l}\text { Évaluation de la satisfaction de l'établissement } \\
\text { Conditions essentielles pour réussir un établissement } \\
\text { Projets d'avenir }\end{array}$ \\
\hline
\end{tabular}

Le montage financier le plus couramment utilisé pour acquérir l'exploitation, soit pour $43 \%$ de l'échantillon (13/30), comprend un apport financier personnel plus un emprunt à une institution financière plus une subvention ou une autre source de financement habituellement fournie par la famille immédiate.

La répartition des succès, insuccès et en difficulté ne permet pas de voir que certaines combinaisons pourraient assurer d'emblée un meilleur succès aux entreprises; cependant, un grand nombre d'entreprises ayant cessé les opérations avaient un apport personnel faible et n'avaient pas obtenu de subventions à l'établissement.

\section{Un enchaînement de facteurs clés}

Parmi les facteurs déterminants, c'est-à-dire ceux qui nous ont semblé avoir une incidence directe sur le résultat, on note d'abord la dynamique interne entre les co-exploitants. Chez les cas de succès, nous sommes en présence d'un projet d'installation vécu et exprimé comme étant un projet de couple, reflétant des valeurs communes et où les démarches ont été faites à deux. Les co-exploitants arrivent à un partage des tâches en complémentarité selon leurs forces, intérêts et compétences, et démontrent une polyvalence et une flexibilité. C'est un facteur ayant un effet très positif au pré-démarrage et qui s'est confirmé 
comme déterminant au démarrage chez la majorité des entreprises encore en opération. Ce phénomène ne s'est pas manifesté chez les insuccès.

La dynamique externe entre l'exploitant et le vendeur de la ferme a aussi été un facteur déterminant qui s'est manifesté surtout sous la forme de parrainage au pré-démarrage. Certains vendeurs «sans relève familiale» ont noué des liens affectifs avec le nouveau venu, le considérant même comme un enfant adoptif, phénomène déjà relevé par Alice Barthez dans ses travaux. Dans bon nombre de cas, on reconnaît une logique patrimoniale derrière les signes laissés par les vendeurs ou les locateurs qui accompagnent les nouveaux exploitants. L'entraide et le soutien financier de la famille immédiate ont aussi été déterminants et ce, autant au pré-démarrage et qu'au démarrage. Ce soutien concerne autant les cas de succès que les insuccès et ceux en difficulté. L'entraide s'est manifestée par de l'apport de main-d'œuvre occasionnelle, mais aussi sous forme d'appui moral. D'autre part, le soutien financier direct a pris diverses formes telles le don, le prêt à des conditions avantageuses, le cautionnement auprès des institutions financières.

Le support professionnel fourni tant par les conseillers agricoles que par les agriculteurs d'expérience a été un élément déterminant, particulièrement en période de pré-démarrage. Il s'agit ici d'initiatives de conseillers agricoles ou d'agriculteurs qui ont joué un rôle d'experts par un apport de connaissances spécialisées, mais aussi un rôle très important de référence vers des sources et réseaux d'information pertinents. Une fois l'installation réalisée, ce sont plutôt les nouveaux exploitants qui vont se former une équipe permanente d'experts avec qui ils développent une relation de confiance et qu'ils vont consulter ponctuellement et régulièrement.

Trois autres facteurs se sont avérés déterminants ou ayant eu un effet très facilitant, soit la capacité à aller chercher des ressources financières, le choix de la région et le choix de la production. C'est de loin le premier facteur qui est le plus important parce qu'il peut avoir un impact majeur sur la réalisation du projet. La moitié des entreprises qui ont réussi démontrent cette capacité, voire un acharnement à obtenir et à varier leurs sources de financement. Quant au choix de la région ou de la production, chez la majorité des succès, on constate une capacité de combiner des raisons affectives et pragmatiques.

\section{Le support professionnel fourni tant par les conseillers agricoles que par les agriculteurs d'expérience a été un élément déterminant, particulièrement en période de pré-démarrage.}

Cinq autres facteurs ayant joué un rôle déterminant ou très facilitant au démarrage sont majoritairement présents dans les cas de succès et quasi inexistants dans les cas d'insuccès et en difficulté. Ce sont : la qualité des compétences managériales, la maîtrise technique de la production, la mise en place de stratégies de réussite, la capacité de s'ajuster aux moments critiques ou de redresser une situation problématique et l'implication dans les réseaux socioprofessionnels. Les deux premiers ne se retrouvent dans aucun cas d'insuccès ni aucune entreprise en difficulté, et les deux derniers, dans trois entreprises seulement. La capacité de s'impliquer professionnellement est, quant à elle, présente chez la majorité des entreprises à succès et quasi inexistante dans les deux autres catégories. Dans un autre ordre d'idées, certains obstacles ont eu un effet direct (blocages) ou indirect (freins) sur le déroulement des affaires de l'entreprise. Ils touchent majoritairement les cas d'insuccès et les cas en difficulté, nous les avons regroupés dans le tableau 4.

\section{Des mythes à déconstruire et des ponts à bâtir}

Outre les tendances observées dans la réussite ou l'échec du démarrage d'entreprises agricoles hors du cadre du transfert familial, les résultats de cette recherche permettent de lever le voile sur les réalités vécues par cette population. Ils permettent aussi de faire prendre conscience qu'un certain nombre de croyances et de mythes partagés autant par les agriculteurs que par les intervenants ne tiennent plus. Les huit mythes que nous avons dégagés se regroupent en deux catégories: les uns concernent les caractéristiques des individus et les autres, la vision de leur projet d'établissement.

Mythe 1. La relève non familiale est composée de " gens de la ville ». L'analyse de notre échantillon montre que, sur l'ensemble des répondants, une forte proportion est de milieu rural et plus encore, d'origine agricole : 20 étaient enfants d'agriculteurs dont 9 hommes et 11 femmes. Diverses raisons expliquent pourquoi ces enfants d'agriculteurs n'ont pas été la 
relève désignée : moment non propice, préférence des parents vers d'autres enfants plus âgés, mésentente avec des membres de la famille, incapacité physique temporaire, etc. Ceux qui ne sont pas d'origine agricole directe ont tous, de près ou de loin, des grands-parents, des oncles qui les ont initiés aux plaisirs de travailler la terre ou de prendre soin d'animaux.

Tableau 4 - Synthèse des facteurs caractérisant l'établissement hors cadre familial

\begin{tabular}{|c|c|}
\hline Facteurs déterminants et très facilitants & Blocages et freins \\
\hline $\begin{array}{l}\text { - Dynamique interne entre les co-exploitants } \\
\text { - Sumplémentarité des tâches, complicité) } \\
\text { - Soutiens de la famille immédiate } \\
\text { - Support professionnel (conseillers et producteurs } \\
\text { agricoles } \\
\text { - Implication dans les réseaux socioprofessionnels } \\
\text { - Capacité à aller chercher des fonds et à varier les } \\
\text { - } \text { sources de financement du projet } \\
\text { la réalisation du projet } \\
\text { - Degré élevé de compétence conjuguant à divers } \\
\text { degrés au moins } 2 \text { éléments tels origine agricole }+ \\
\text { formation spécifique + expérience dans le secteur } \\
\text { choisi. }\end{array}$ & $\begin{array}{l}\text { - Manque d'expérience dans la production } \\
\text { - Manque de formation en gestion (aspects financiers, } \\
\text { organisation du travail, comptabilité) } \\
\text { - Stratégies financières déficientes } \\
\text { - Montage financier inadéquat } \\
\text { - Absence de marge de manœuvre en situation de crise } \\
\text { - Conditions fluctuantes du marché } \\
\text { - Projet d'établissement non partagé par le conjoint } \\
\text { - Support professionnel déficient de l'intégrateur. }\end{array}$ \\
\hline
\end{tabular}

Mythe 2. L'installation fait suite à une décision prise sur un coup de tête. Une seconde réalité qui nous a été révélée est le fait que l'installation n'est pas le fruit d'une idée surgie spontanément. En effet, l'idée de démarrer une entreprise agricole a pris racine à l'adolescence dans la grande majorité des cas, voire pendant l'enfance. Cette réalité est aussi présente même chez ceux qui ont connu un échec. Plusieurs en parlent comme le rêve de leur vie, un besoin d'accomplissement. Ils ne peuvent s'imaginer faire autre chose. Certains disent même avoir l'agriculture dans le sang. Majoritairement, ce sont des hommes où l'idée est présente depuis longtemps et chez plusieurs couples, l'un a communiqué à l'autre son rêve et ainsi, le projet est devenu collectif. Cette idée peut avoir été encouragée et stimulée par les parents déjà agriculteurs ou enfants d'agriculteurs ou tout autre membre de la parenté.

Mythe 3. La relève non familiale ne connaît rien à l'agriculture. L'échantillon révèle que la relève non familiale est nombreuse à avoir une formation spécialisée en agriculture : les trois-quarts ont au minimum une formation collégiale spécialisée en agriculture et quatre, une formation universitaire. Dans cinq cas, les deux co-exploitants avaient une formation agricole. Ils sont convaincus de la nécessité d'acquérir une formation initiale en agriculture pour démarrer lorsqu'on n'est pas originaire du milieu. En revanche, dans $60 \%$ des entreprises en difficulté ou en échec, les exploitants étaient sans formation en agriculture.

Mythe 4. Les jeunes n'ont jamais mis les pieds sur une ferme. L'établissement n'est pas réalisé directement après la formation. Il y a généralement une période plus ou moins longue sur le marché du travail. En effet, excepté quelques cas où l'établissement s'est réalisé sans décalage à la suite des études, tous les autres ont plusieurs années d'expérience de travail agricole en lien plus ou moins direct avec leur formation. Certains étaient techniciens en alimentation des animaux, en horticulture, d'autres des agronomes. C'est souvent dans le cours de leur travail que le projet se précise et que des démarches concrètes sont réalisées. Une expérience concrète en agriculture est souvent la source d'inspiration de l'idée du projet d'établissement. Excepté dans trois entreprises, les exploitants avaient une expérience plus ou moins directe avec le projet, expérience variant entre quelques mois et une quinzaine d'années. 
Mythe 5. Les jeunes ne savent pas dans quoi ils s'embarquent. Lorsque l'idée commence à prendre forme, que la production est choisie mais que l'expérience de la production n'a pas été acquise, les jeunes commencent diverses démarches et s'organisent un stage informel avec un autre producteur ou procèdent à des expérimentations. C'est le cas de presque la moitié de l'échantillon. Pour les futurs exploitants, c'est une sorte de test pour confirmer leur désir de s'établir dans le métier et dans la production choisie, mais aussi un moyen d'acquérir une expérience pratique. Les personnes approchées qui leur transmettent généreusement leurs savoirs dans les stages sont des producteurs plus chevronnés, amis ou non, ayant une expertise dans la production. Notons que cette pratique est surtout le lot des jeunes qui se lancent dans les productions en émergence, qui ne sont pas toujours aussi bien documentées et connues par les conseillers agricoles.

Mythe 6. Les jeunes ne savent pas ce qu'ils veulent. Les jeunes rencontrés avaient une vision claire de leur projet qui s'insérait dans un projet plus général de vie. Les valeurs invoquées tournent autour de l'idée que l'agriculture est un projet de couple et un style de vie, une voie idéale pour la conciliation travail-famille, s'apparentant ainsi à ce que révélaient nos travaux sur la relève familiale. Ils sont à la recherche d'un mode de vie : travailler ensemble, vivre à la campagne, vivre de l'agriculture.

Mythe 7. Un projet de "gentleman farmer». Une étude exhaustive reste à faire pour bien comprendre la problématique du travail à temps partiel et la déconstruction d'une vision négative des producteurs qui s'établissent à temps partiel (souvent identifiés à des agriculteurs « du dimanche »). Dans notre échantillon, le travail à temps partiel n'est pas un choix mais une nécessité. Dès la planification du projet, la plupart des couples prévoient augmenter les revenus de la ferme de façon à obtenir des ressources qui permettront à la famille de subvenir à ses besoins.

Mythe 8. Les jeunes ne tiendront pas le coup. Une obstination et un acharnement à réussir l'installation se dégagent de l'ensemble des personnes rencontrées, même chez celles qui ont dû abandonner. Qui plus est, les exploitants sont conscients qu'ils sont à bâtir leur patrimoine. Plus de la moitié des couples rencontrés désirent laisser un héritage à leurs enfants, s'inscrivant ainsi dans la même logique patrimoniale qui caractérise les installations effectuées dans le cadre du transfert familial parents-enfants.

\section{Pistes de réflexions et d'actions : viser une reconnaissance politique et socioprofessionnelle.}

Évidemment, il serait important de mettre à l'épreuve nos conclusions sur une plus vaste population. Un portrait exhaustif permettrait ainsi d'obtenir une vue d'ensemble du phénomène d'établissement hors cadre familial pouvant guider les acteurs du milieu vers des politiques, programmes et interventions mieux ciblés, notamment en ce qui concerne l'aide et le soutien à l'établissement. Cependant, suite à nos résultats, quelques pistes de réflexions s'imposent.

- Il apparaît nécessaire de viser à une reconnaissance socioprofessionnelle et politique de ce groupe et ce, par les diverses instances tels les organismes gouvernementaux, les organisations agricoles syndicales, les institutions financières et les conseillers agricoles de divers milieux. Dans le contexte où le Québec veut faire cesser la diminution inquiétante du nombre de fermes, il y a nécessité d'établir une politique à l'établissement qui fait de la place à l'établissement graduel, à temps partiel; c'est une réalité incontournable empruntée par les jeunes couples pour s'intégrer en agriculture. De fait, qu'il s'agisse uniquement de l'aide financière à l'établissement accordée actuellement à ceux qui démarre, celle-ci nous apparaît être un obstacle plutôt qu'un facteur facilitant, une aide au transfert de ferme familiale plutôt qu'une aide à l'installation de la relève agricole.

- Il serait aussi souhaitable d'établir une porte d'entrée unique pour l'accompagnement des jeunes. Nous avons vu que l'accompagnement professionnel s'est avéré essentiel, mais il est dispersé et surtout, mal connu des jeunes. Il y a urgence à bâtir des ponts entre les réseaux voués au développement des territoires et les dispositifs et organismes agricoles construits autour des secteurs de production. Tout comme il semble impératif de reconnaître et de développer les compétences sociales requises par les conseillers appelés à intervenir dans des situations de transfert et d'établissement en agriculture.

- Il devrait y avoir un encouragement et un encadrement au parrainage et ce, en termes de valorisation 
financière et professionnelle de la contribution des producteurs d'expérience dans le processus d'apprentissage des nouveaux agriculteurs. De plus, il faudrait instaurer une profonde réflexion sur les ambiguïtés de l'installation par voie de l'intégration (c'est-à-dire par voie de lien contractuel unissant un fournisseur d'intrants et un agriculteur). S'établir par voie d'intégration s'est avéré une nécessité pour certains jeunes. Cependant, cette avenue s'est révélée «noire ou blanche ». Peu de jeunes étaient au courant de toutes les implications des contrats qu'ils avaient signés et, compte tenu de cette vulnérabilité, un mécanisme d'accompagnement professionnel serait nécessaire.

- Finalement, à côté de la politique jeunesse adoptée par le MAPAQ en 2005, qu'en est-il de l'ensemble de la politique agricole au regard de la durabilité du secteur agricole ? Espérons que la Commission québécoise sur l'agriculture et l'agroalimentaire nous apportera quelques réponses.

\section{Tableau 5 - « Ce qu'ils nous ont dit.... »}

\section{Sur les conditions de succès :}

« Pour réussir ça m'a pris un petit peu de naïveté, bien de la folie et de la persévérance » (Un agriculteur de la Montérégie)

\section{Sur le rôle crucial du vendeur :}

«L'ancien propriétaire avait des enfants et aucun ne désirait reprendre la ferme. [...] Je lui ai dit en souriant : la seule condition pour que j'achète c'est que vous restiez comme consultant pendant un an avec nous. Il était content parce qu'on était comme sa relève $[\ldots]$ on est devenus complices [...] j'en ai eu assez pour naviguer tout seul». (Un co-exploitant du Bas Saint-Laurent)

\section{Sur la subvention à l'établissement:}

«La prime : ce n'est pas assez ouvert aux personnes qui veulent s'établir. Le titre est mal défini à mon avis; c'est plus une prime aux établis et aux parents qu'une prime à l'établissement. [...] Il faudrait que les jeunes puissent avoir accès à du financement plus abordable sans avoir besoin d'être établis. Un bon plan d'affaires serait suffisant.» (Un co-exploitant du Centre du Québec)

\section{Sur le support familial :}

«Mon père a tout le temps rêvé d'avoir une ferme. Quand il a vu que je voulais m'établir en agriculture, il a tout fait pour m'aider. » (Un co-exploitant du Centre du Québec)

\section{Sur l'amour porté à leur métier et à leur choix de vie :}

"Quand on a acheté, au printemps, trois semaines ou un mois après, c'était assez beau pour se promener dans les champs. J'ai fait le tour de mes champs à pied et ça ne s'explique pas ce que j'ai vécu. C'était mes terres à moi et j'étais chez moi. Je m'en rappellerai toute ma vie. [...] Quand c'est chez toi, ce n'est pas pareil [...] t'essaies d'améliorer ta terre, ça pousse bien, tu ne peux pas faire autrement qu'être content. » (Un co-exploitant en production laitière, Centre du Québec)

\section{Notes et références}

1 Commission sur l'avenir de l'agriculture et de l'agroalimentaire du Québec (CAAAQ) (2007). Agriculture et agroalimentaire, choisir l'avenir, document de consultation, janvier, p. 37.

2 Statistique Canada, Recensement agricole de 2006, [En ligne] http://www41.statcan.ca/0920/ceb0920_000_f.htm

3 MAPAQ (2002). Profil de la relève agricole au Québec. 2000, Québec, Gouvernement du Québec, Ministère de l'Agriculture, des Pêcheries et de l'Alimentation du Québec, Direction du développement de la main-d'œuvre du bio-alimentaire.
4 Barthez, A. (1999). «Installation hors du cadre familial et relation d'adoption ", Économie Rurale, n 253, p. 15-20; Ponchelet, D. et P. Mundler (1999). « Agriculture et mobilité sociale. Ces agriculteurs venus d'ailleurs », Économie rurale, $\mathrm{n}^{\mathrm{o}} 253$, p. 21- 27.

5 Parent, D., Ouellet, É. et J.-P. Perrier en collaboration avec Marie Gagné (2004). Établissement en agriculture de la relève non familiale: trajectoires et facteurs de succès et d'insuccès, TRAGET Laval et Université Laval, février.

6 Parent, D., Jean, B. et M. Simard (2000). Transmission et maintien de la ferme familiale: analyse des facteurs et stratégies d'établissement de la relève agricole familiale via les situations d'insuccès, Québec, Université Laval et Agri-Gestion Laval, mars. 


\section{Publicité}

Maîtrise en gestion de projet 